\title{
Konstellative Anthropologie Zum Bild des Menschen im alten Ägypten
}

\author{
Jan Assmann
}

\section{Menschenbild und Totenglauben}

Fast alles, was wir über die altägyptische Kultur wissen, stammt aus Gräbern. Kein Wunder, daß der Tod, die Auseinandersetzung mit ihm, die rituellen Anstalten $\mathrm{zu}$ seiner Bearbeitung, die Monumente zu seiner Überwindung durch Verewigung uns heute als die Mitte der altägyptischen Kultur erscheint. ${ }^{1}$ Vielleicht handelt es sich nur um eine optische Täuschung, die dem Zufall der Überlieferung verdankt wird? Ägyptische Gräber sind jedoch etwas ganz anderes als das, was wir uns unter einem Grab vorstellen. Sie sind Speicher des Lebens, denn die Ägypter wollten in Gestalt von Wanddarstellungen, Inschriften, Beigaben und Literatur so viel Leben wie möglich ins Grab mitnehmen. So geben diese Gräber einen ungewöhnlich reichen und weiten Ausblick auf die ägyptische Kultur, und wir müssen davon ausgehen, daß der zentrale Ort, den der Tod nach diesen Zeugnissen in dieser Kultur eingenommen zu haben scheint, dem realen Befund sehr nahe kommt. Das gilt auch für den ägyptischen Begriff von Person und Selbst. Wie sich die alten Ägypter den Menschen vorstellten, das erfahren wir vor allem aus den Bildern und Texten, die es mit den Verstorbenen und den Riten ihrer Wiederherstellung als Person im Diesseits und Jenseits zu tun haben.

In den Riten des Totenkults vollzieht der Verstorbene den Osiris-Mythos nach. Der Gott Osiris ist das mythische Ur- und Vorbild menschlicher Todesüberwindung. Die Bilder und Texte des Totenkults orientieren sich an den Szenen des Osirismythos. Dieser Mythos wird in der altägyptischen Überlieferung, soweit sie uns erhalten ist, nie zusammenhängend erzählt, sondern liegt einer großen Masse religiöser Texte als gemeinsamer Anspielungsfundus zugrunde. ${ }^{2}$ Wir müssen und können ihn aus diesen Anspielungen und Voraussetzungen erschließen - nicht als eine zu-

Zur ägyptischen Totenreligion und ihrer Ausstrahlung auf die ägyptische Kultur insgesamt vgl. Assmann, Tod und Jenseits. Die folgende Darstellung des altägyptischen Menschenbildes beruht weitgehend auf dieser Publikation. Zum ägyptischen Menschenbild vgl. ferner Donadoni, Der Mensch im Alten Ägypten.

2 Zu diesem Problem s. Assmann, Verborgenheit des Mythos. 
sammenhängende Geschichte, sondern eher als eine Szenenfolge. Die einzigen Texte, in denen uns der Stoff in fortlaufender Erzählung vorliegt, sind in griechischer Sprache verfaßt, von Diodor ${ }^{3}$ und vor allem von Plutarch. ${ }^{4}$ Aber gerade in ihrer narrativen Kohärenz, ihrer Bemühung um eine einheitliche, sinn- und spannungsvolle Geschichte scheinen sich diese Autoren besonders weit von der ägyptischen Form des Mythos zu entfernen.

Der Mythos spielt nach dem Tod des Osiris und entfaltet seine Szenenfolge erst von diesem tragischen und katastrophischen Ausgangspunkt aus. Aber er setzt natürlich eine Vorgeschichte voraus, ohne die er gar nicht verständlich ist. Wie kommt es zu diesem Tod eines Gottes? Dieser Tod war kein natürlicher; Osiris war, als die Götter noch auf Erden herrschten, König Ägyptens und wurde von seinem Bruder Seth erschlagen, der den Thron an sich reißen wollte. Damit kommt der Tod in die Welt und konfrontiert die Götter mit einem großen Problem. Auf dieser Vorgeschichte aufbauend, sie voraussetzend aber nirgends genauer schildernd, entfaltet sich der eigentliche Mythos in der Form einer Szenenfolge.

Was diese Szenenfolge angeht, fällt sofort eine Zweiteilung ins Auge: Sie ist um zwei zentrale Bilder herum organisiert. Das eine betrifft die Wiederherstellung des Körpers, dessen Todeszustand als Zergliederung und Zerrissenheit dramatisiert wird. Hier geht es um das Sammeln und Zusammenfügen der einzelnen Glieder und ihre Beweinung und Beseelung bis hin zu einer neuen Form leiblicher Ganzheit und Unversehrtheit. Das andere Bild betrifft die Wiederherstellung der Sozialbeziehungen des Toten, die Aufhebung seiner totalen Isolation, seines Herausgefallenseins aus allen sozialen Lebensbindungen, das als ein totaler Verlust von Status, Würde, Ehre, Prestige dramatisiert wird. Beide Aspekte beziehen sich auf die Ausgangssituation des Toten, bei der die Riten der Todesbehandlung ansetzen. Beide werden sie ins Extreme übersteigert oder „dramatisiert": der leblose Körper wird als zerrissen und zergliedert dargestellt, und die Trennung von den Lebenden als eine Art von Schande und Ehrverlust. Je tiefer der Ausgangspunkt, desto höher der Zielpunkt der rituellen Umwandlung. Daher wird der Tod in den ägyptischen Totentexten oft vollkommen schonungslos und in krassesten Farben dargestellt. Beide Todesbilder verweisen auf die Gegenbilder des Lebens: als leiblichen und sozialen Zusammenhang.

\footnotetext{
3 Diodorus Siculus, Bibliotheca hist. I, 11-27.

4 De Iside et Osiride.
} 
Diese Zweiteilung ist für das ägyptische Menschenbild und insbesondere die ägyptische Seelenvorstellung fundamental. Der erste Schritt zur „Behandlung“ des Todes bestand für die Ägypter darin, den Komplex Tod aufzuteilen in einen körperlichen und einen - nicht etwa "seelischen", wie wir vielleicht erwarten würden, sondern vielmehr - sozialen Aspekt und beiden Aspekten eine jeweils andere Behandlung angedeihen zu lassen. Daran schließt sich eine nicht minder merkwürdige Beobachtung. Die Behandlung des körperlichen Todesaspekts war eine vornehmlich weibliche, die Behandlung des sozialen Aspekts eine vornehmlich männliche Aufgabe. Um die Wiederherstellung des Leibes, das Einsammeln und Zusammenfügen der Glieder, ihre Beweinung und Beseelung, sehen wir Isis und Nephthys bemüht, denen der Gott Anubis assistiert, und die Wiederherstellung der Ehre des Toten und seine Resozialisation in der Götterwelt liegt in den Händen des Horus, dem der Schreibergott Thot, die vier "Horussöhne" und wiederum Anubis beistehen. Der Zweiteilung des Todeskomplexes entspricht also eine eindeutige Geschlechterdifferenzierung in der Rollenverteilung der Todesbehandlung.

In den Klageliedern der Isis und Nephthys ${ }^{5}$ geht es einerseits um Gefühle der Liebe, Sehnsucht und Trauer, und andererseits um den Körper des Geliebten, den sie von Kopf bis Fuß beschreiben, um ihn im Medium des Textes wieder zusammenzusetzen. Der Körper erscheint hier, mit dem treffenden Ausdruck von E. Brunner-Traut, wie eine „Gliederpuppe "66, aber nicht, weil die Ägypter unfähig waren, ihn als eine organische Ganzheit zu begreifen, sondern weil es ihnen auf die Verbindung der Teile zu einem neuen Ganzen ankam, auf das Prinzip der Konnektivität, das den in seine Einzelglieder zerfallenen Körper wieder zu einer Ganzheit zusammenzufügen vermag. In den Augen der Ägypter erfüllt beim lebenden Körper das Blut die Funktion des konnektiven Prinzips. Mit diesem Konzept kommen sie bereits 3000 Jahre vor Harvey erstaunlich nahe an eine Theorie des Blutkreislaufs heran. Das Herz pumpt das Blut durch die „Gefäße“ und „verknotet“ auf diese Weise die Glieder zum Körper. Wenn das Herz aufhört, zu schlagen, zerfällt der Körper wieder in seine Einzelglieder. Dieser Zustand wird im Todesbild der Zerrissenheit mythisch dramatisiert. Jetzt muß die Funktion des Herzens und des Blutes mit anderen Mitteln wahrgenommen werden, mit den Mitteln der Magie, durch Amulette, Symbole und Sprüche.

Bei dieser leiblichen Wiedervereinigung bleiben die Ägypter aber nicht stehen. So wie für uns die Person aus Leib und Seele besteht, so be-

Kucharek, Klagelieder.

Brunner-Traut, Gliederpuppe, $8 \mathrm{ff}$. 
steht sie für den Ägypter aus dem Zusammenwirken leiblicher und sozialer Konnektivität. Der Mensch lebt in einer Leibsphäre und einer Sozialsphäre. In beiden Sphären kommt es auf die konnektiven Kräfte an, die die Teile zur Ganzheit zusammenfügen. In der Leibsphäre ist das die Aufgabe der liebenden Gattin, in der Sozialsphäre die des liebenden Sohnes. Die Sohnesliebe ist bei der Behandlung des Todes genauso wichtig wie die Gattenliebe. Der Mythos gibt diesen Affekten eine gültige Form, das Ritual bringt sie in seiner Inszenierung als konnektive Kräfte zum Tragen. Beide Affekte haben die Kraft, die Schwelle zwischen Leben und Tod, Diesseits und Jenseits, zu überspannen und den Toten aus dem Todeszustand herauszuholen: nicht zurück ins irdische Leben, wie es Orpheus im griechischen Mythos mit Eurydike anstrebte, sondern vorwärts in ein dem Tode enthobenes Dasein in der Götterwelt als ,verklärter Ahnengeist" (ägyptisch $3 \not h / \bar{a} c h$ ).

So wie Isis und Nephthys zusammen mit Anubis den Toten in seiner leiblichen Ganzheit wiederherstellen, so stellt Horus ihn als soziale Person wieder her. Hier geht es darum, ihn aus seiner Isolation zu befreien und ihm aus der Tiefe seiner Entehrung und Entwürdigung, in die Seth ihn durch die Schändung der Leiche gestoßen hat, zur völligen Rehabilitation zu verhelfen, seine Ehre wiederherzustellen, ihm in der Götterwelt Respekt zu verschaffen und ihn in seine herrscherlichen Rechte wiedereinzusetzen. Vor allem aber kommt es darauf an, dem Toten gegenüber dem Tod, seinem Mörder, zum Recht zu verhelfen, das heißt Osiris „gegen“ Seth zu „rechtfertigen“. Diese „Rechtfertigung gegen" den Tod bildet den entscheidenden Schritt der Todesüberwindung. Dafür muß Seth vor Gericht gestellt und verurteilt werden. Der Thron des Osiris fällt seinem Sohn Horus zu, Osiris wird Herrscher der Unterwelt. Damit ist die Welt wieder in Ordnung, der Tod geheilt.

\section{II. „Ba“ und „Ka“, Leibsphäre und Sozialsphäre}

In diesen mythischen Bildern und Riten wird ein Menschenbild greifbar, das nicht nur zwischen Körper und Seele unterscheidet, sondern dem Menschen zwei verschiedene „Seelen“ zuschreibt, die „Ba“" und „Ka“ genannt werden. Der Sinn dieser Unterscheidung läßt sich als „Körperseele“ und „Sozialseele“ verstehen. ${ }^{7}$ Der Ba, die Körperseele, beseelt den Kör-

7 Vgl. Assmann, Tod und Jenseits, 116ff. Den älteren Studien zu Ka (Greven, Ka; Schweitzer, Wesen des Ka; Kusber, Ka; Borioni, Ka) und Ba (Wolf-Brinkmann, $\mathrm{Ba}$; Zabkar, Ba-Concept) ist der Sinn dieser Zweiteilung entgangen; sie kommen daher zu sehr anderen Ergebnissen. 
per zu Lebzeiten und trennt sich von ihm nach dem Tod, um zum Himmel aufzusteigen, hält aber die Verbindung zum mumifizierten Leichnam aufrecht, auf dem er sich allnächtlich niederläßt. Der Ba wird als Vogel mit Menschenkopf dargestellt: der Vogelleib symbolisiert Freizügigkeit und Himmelsaufstieg, der Menschenkopf das Prinzip der Individualität, der physiognomischen Unverwechselbarkeit. Auch wenn der Ba nach dem Tode das Prinzip der Freizügigkeit darstellt, und alle Weltbereiche Himmel, Erde und Unterwelt - durchschweift, bleibt er doch mit dem Körper verbunden und ist auch in seiner Freizügigkeit Medium körperlicher, z.B. sexueller Erfahrungen.

Der Ka wird mit einem Zeichen geschrieben, das ein Paar umarmend vorgestreckter Arme darstellt. Der $\mathrm{Ka}$, so wird es in vielen Texten beschrieben, geht in Form einer Umarmung vom Vater auf den Sohn über. So umfaßt zum Beispiel der Schöpfergott Atum das aus ihm entstandene Zwillingspaar Schu und Tefnut:
Atum Cheprer,
du bist aufgegangen auf dem Benben
im Phönixhaus in Heliopolis.
Du hast ausgespien als Schu
und ausgehustet als Tefnut.
Du hast deine Arme um sie gelegt als die Arme des Ka,
damit dein $\mathrm{Ka}$ in ihnen sei.
Atum, mögest du dir deine Arme auch um NN geben,
(um dieses Bauwerk, um diese Pyramide) als Arme des Ka, damit der Ka des NN in ihm sei,
fest für immer und ewig. ${ }^{8}$

Von dieser Umarmung erzählt auch das „Denkmal memphitischer Theologie“, ein Text des 8.Jh.s v.Chr., der die Stadt Memphis als die Königsstadt Ägyptens herausstellt. ${ }^{9}$ Osiris ist von seinem Bruder Seth erschlagen worden. Dem Sohn Horus gelingt es aber, dem Mörder das Königtum zu entreißen. Osiris wird bestattet, und Horus besteigt den Thron „in den Armen" des toten Vaters:

So geriet Osiris in die Erde in der Königsburg auf der Nordseite dieses Landes, zu dem er gelangt war.

Sein Sohn Horus erschien als König von Ober- und Unterägypten in den Armen seines Vaters Osiris inmitten der Götter, die vor ihm und hinter ihm waren. ${ }^{10}$

Sethe, Pyramidentexte, $\S \S 1652 \mathrm{f}$.

S. dazu Sethe, Dramatische Texte; Junker, Götterlehre; ders., Politische Lehre.

10 Sethe, Dramatische Texte, 76 f. 
In der Ka-übertragenden Umarmung wird ein Bund zwischen Diesseits und Jenseits, Lebenden und Toten, gestiftet, der die Grundlage der ägyptischen Gesellschaft bildet. Jeder Pharao ist Horus und steht mit dem Jenseits in Verbindung, als hinterbliebener "Sohn" sowohl der gesamten Reihe seiner Amtsvorgänger bis zurück in grauste Vorzeit, als auch der gesamten Götterwelt, der er im Kult als seinen Vätern und Müttern gegenübertritt. Das Band, das der Ka zwischen totem Vater und hinterbliebenem Sohn über die Todesschwelle hinweg knüpft, bindet und trägt die ganze ägyptische Religion, Kultur und Gesellschaft.

Durch die Sozialseele, die der Sohn vom Vater erbt und an seine Kinder weitergibt, ist der einzelne in die Gemeinschaft eingebunden. $\mathrm{Zu}$ dieser Gemeinschaft gehören vor allem die Toten. Die Urzelle der Gemeinschaft bildet die Verbindung von totem Vater und hinterbliebenem Sohn, deren mystisches Band über die Todesschwelle hinweg die Kaübertragende Umarmung symbolisiert. ${ }^{11} \mathrm{Da}$ hier von Vater und Sohn die Rede ist, unter scheinbarer Absehung von Mutter und Tochter, soll uns nicht täuschen: das Ka-Konzept ist im gleichen Sinne verallgemeinerbar wie der von Sigmund Freud postulierte Ödipus-Komplex. Der Ka ist nicht nur das ägyptische Äquivalent zu der von Freud herausgestellten patri-ödipalen Tiefenstruktur der menschlichen Seele, er stellt auch das genaue Gegenteil der Freudschen Konzeption dar. Beruht der ÖdipusKomplex auf der Rivalität zwischen Vater und Sohn, woraus sich als Grundbedingung eines gelingenden Lebens die Ablösung des Sohnes vom Vater ergibt, so beruht der Ka auf dem Gedanken einer wechselseitigen Angewiesenheit. Der Sohn ist nichts ohne den Vater, der Vater nichts ohne den Sohn:

„Āch" ist ein Vater für seinen Sohn,

"āch“" ist ein Sohn für seinen Vater. ${ }^{12}$

„Āch“ ist ein Schlüsselwort der ägyptischen Totenreligion. Es bezeichnet eine heilskräftige Wirksamkeit über die Todes- bzw. Diesseitsschwelle hinweg. Auf diesem Vater-Sohn-Bündnis beruht die ägyptische Kultur. Dieses Bündnis wird in der mystischen $\mathrm{Ka}$-Umarmung symbolisiert und besiegelt. Wie bei Freud Vatermord und Bruderbund, so gehören bei den Ägyptern Brudermord und Vaterbund zusammen. Seth, der Mörder des Osiris, ist dessen Bruder; Horus, der Rächer des Osiris, ist dessen Sohn. So wie der Bruderbund das Prinzip einer Gemeinschaft darstellt auf der

11 S. dazu Assmann, Bild des Vaters, besonders $115 \mathrm{ff}$, sowie ders., Tod und Jenseits, $116 \mathrm{ff}$. Zur Bedeutung von ' $h$ /ăch s. zuletzt Jansen-Winkeln, ,Horizont‘.

12 Liturgie NR.1.1 in: Assmann, Totenliturgien II, 61ff Verse 13-14, 80 §5. Vers 1 kommt bereits in einer Grabinschrift des Alten Reichs vor, s. aaO 80 Anm.57. 
Grundlage einer ,horizontalen“ Solidarität zwischen Gleichen, so fordert der Vaterbund die „vertikale“ Solidarität zwischen Ungleichen: Höheren und Niederen in der Sozialdimension, Toten und Lebenden in der Zeitdimension. ${ }^{13}$

\section{Das Herz: Einer lebt, wenn der andere ihn leitet}

Im Rahmen dieses Modells erscheint nichts abwegiger und undenkbarer als die Freudsche Vatersemantik mit Kastrationsangst, Triebverzicht, Vatermord und Vaterkult, d.h. die ganze ödipale Struktur, die Freud als Grundstruktur jeder menschlichen Seele voraussetzte. ${ }^{14}$ Und doch lässt sich der Ka durchaus als ein Über-Ich im Freudschen Sinne interpretieren, das dem einzelnen von seinem Vater bzw. seinen Vätern her zukommt und das er seinen Kindern weitergibt. Auch in Ägypten erscheint der Vater als der zentrale kulturelle Normensender, und diese Normen fordern Triebverzicht und Selbstzurücknahme. Den Ägyptern aber erscheint die in diesen Normen vermittelte Kultur nicht als Zwangsjacke, sondern als das lebenspendende Prinzip schlechthin.

Für die Ägypter beginnt das Leben nicht mit der Geburt, sondern mit der Erziehung. „Der eine lebt, wenn der andere ihn leitet“, lautet das Sprichwort. ${ }^{15}$ Allein kann der Mensch nicht leben, er bedarf des anderen. Zum Leben gehören mindestens zwei. In die gleiche Richtung weist auch eine Sentenz, die in einer Lebenslehre überliefert ist: „Ein Mensch entsteht, wenn er von Menschen umgeben ist. Er wird ehrfürchtig gegrüßt um seiner Kinder willen. “16 Ein Mensch entsteht nach Maßgabe seiner konstellativen Entfaltung in der „Mitwelt“ seiner Familie, Freunde, Vorgesetzten, Abhängigen.

Wie nun der eine erst lebt, wenn der andere ihn geleitet, so gilt aber auch, daß er zu solchem Sich-leiten-lassen erst erzogen werden muß. Durch seine Sozialseele ist der Mensch auf das Leben in Gemeinschaft angelegt und angewiesen, aber diese Anlage bedarf der Ausbildung. Auf der Grundlage unseres „ödipalen“ Menschenbildes würden wir eines der edukativen Hauptziele in der Erziehung zur Selbständigkeit erblicken. Die Ägypter dachten umgekehrt. Selbständigkeit, Autonomie, Durchset-

13

14

15

16
Zum Begriff der ,vertikalen Solidartät“ s. Assmann, Ma'at, $92 \mathrm{ff}$.

Freud, Totem und Tabu.

In einem magischen Text äußert die Göttin Isis diese Sentenz wie ein gängiges Sprichwort. Metternichstele M 50, hg. Sander-Hansen, Metternichstele, 35f, 41; Klasens, Magical Statue Base, 10, 52; Sternberg, Metternichstele, 376.

Lehre des Ani 16.3, Fassung des pLouvre, s. Quack, Lehren des Ani, 285. 
zungsvermögen und Selbstbehauptung würden sie zur rohen Natur rechnen, die es durch Bildung zu überwinden oder überformen gilt in Richtung auf Bindung, Altruismus, Selbstzurücknahme und Einfügung. Solche „Bildung“ ermöglicht es dem einen, sich vom anderen geleiten zu lassen, und ermöglicht dem anderen den geleitenden Umgang. Sitz der konnektiven Tugenden, die dem einzelnen das Leben ermöglichen, indem sie ihn zur Bindung befähigen, ist dasselbe Organ, das ihm auch das Leben ermöglicht, indem es ihn als eine innere Vielheit von Aspekten und Konstituenten organisiert und koordiniert: das Herz. ${ }^{17}$ Von Natur aus ist das Herz der Sitz der Triebe und Leidenschaften. Durch Erziehung und Bildung aber wird es umgeformt in das Sozialorgan par excellence, und zwar als Organ des Verstehens im synchronen Raum der Sprache, der Kommunikation, des Aufeinander-Hörens, und als Organ des Gedächtnisses im diachronen Raum der Erinnerung und des Wartens, in der sozialen Zeit des Füreinander-Handelns. ${ }^{18}$

Dabei zeigt sich, daß der Begriff der Konstellation in einem doppelten Sinn zu verstehen ist, der sich aus der ägyptischen Unterscheidung zwischen einer Sozialsphäre und einer Leibsphäre ergibt. Einerseits bedeutet Leben die Eingebundenheit in soziale Konstellationen, andererseits wird aber auch die Person selbst als eine konstellierte Vielheit gedacht, und so wie einer lebt, wenn er von anderen geleitet wird, so lebt er auch erst, wenn er diese Vielheit seiner inneren Konstituenten und Aspekte zu leiten versteht. Ägyptisch heißt das: ,von seinem Herzen geleitet werden'. Das Herz übt dieselbe Leitung nach innen aus, die ihm durch den anderen von außen zuteil wird. Einer lebt, wenn sein Herz ihm Arme und Beine, Augen und Ohren, Leib und Seele, Denken und Fühlen, Einsicht und Leidenschaften koordiniert. Wenn diese innere Leitung ausfällt, steht es schlimm um den Betroffenen. Daher sagt man zum Toten, wenn man ihn in den Zustand der Lebendigkeit zurückwünscht:

Siehe, dein Herz leitet dich, und deine Glieder gehorchen dir. ${ }^{19}$

Die Führerschaft des Herzens hat also zwei verschiedene Aspekte, die man sorgfältig auseinanderhalten muß: den sozialen Aspekt in der äußeren Sphäre des Lebens und Handelns in der Gesellschaft und den leiblichen Aspekt in der inneren Sphäre körperlicher und geistiger Einheit. Das Herz ist die "Schnittstelle" zwischen der Sozialsphäre und der Leibsphäre. In der leiblichen Sphäre ist der Idealzustand voller Lebendigkeit

17 Brunner, Herz; ders., Das hörende Herz; Assmann, Geschichte des Herzens.

18 Vgl. dazu Assmann, Ma'at, 60ff.

19 Sethe, Urkunden, 519.14, mit vielen Parallelen. 
erreicht, wenn das Herz (a) „lebendig“ oder „wach“, und (b) „auf seinem Platz" ist. Daher muß das Herz (a) stimuliert, aufgeweckt, belebt, und (b) be- und gefestigt werden. Die entsprechenden Gegenzustände sind (a) das „müde Herz" bzw. Herzensmüdigkeit, eine Bezeichnung des Todeszustands, und (b) das losgelöste oder abwesende Herz. In beiden Zuständen, dem der Müdigkeit und dem der Abwesenheit, versagt das Herz in seiner zentralisierenden Funktion, und die Person zerfällt in eine disparate Vielheit. Ein berühmtes Beispiel solchen Personzerfalls bietet die Altersbeschreibung in der Lehre des Ptahhotep:

Gebrechlichkeit ist entstanden, das Greisenalter ist eingetreten,

Schwäche ist gekommen, die kindliche Hilflosigkeit kehrt wieder, die Kraft schwindet, denn müde ist mein Herz.

Der Mund ist verstummt und spricht nicht mehr, die Augen sind trübe, die Ohren sind taub, das Schlafen fällt ihm schwer Tag für Tag; das Herz ist vergeßlich, es erinnert sich nicht mehr an gestern, der Knochen ist krank wegen der Länge < der Jahre>, die Nase ist verstopft, sie kann nicht atmen, denn beschwerlich sind Aufstehen und Niedersetzen.

Das Gute wurde zum Schlechten, jeder Geschmackssinn ist geschwunden.

Was das Alter den Menschen antut: Schlimmes in jeder Weise. ${ }^{20}$

Ein solcher Zerfall personaler Einheit durch Ausfall des Herzens kann aber auch andere Ursachen haben wie z.B. Sehnsucht und Heimweh:

Sieh, mein Herz ist diebisch fortgegangen, es eilt zu dem Ort, den es kennt, es reist stromauf, um Memphis zu schauen. Ich aber, ich sitze (zu Hause) und warte auf mein Herz, daß es mir den Zustand von Memphis sagen könnte.

Kein Auftrag gelingt mehr in meinen Händen: mein Herz ist fortgelaufen von seinem Platz. Komm zu mir, o Ptah, und hole du mich nach Memphis.

$\mathrm{La} ß$ mich dich sehen nach Belieben.

Ich wache, aber mein Herz schläft, mein Herz, es ist nicht in meinem Leib.

Alle meine Glieder sind vom Übel ergriffen: mein Auge zu matt zum Sehen, mein $\mathrm{Ohr}$, es hört nicht, 
meine Stimme ist heiser, alle meine Worte verdreht.

Sei mir gnädig! Gib, daß ich daraus erstehe! $!^{21}$

„Ich wache, aber mein Herz schläft" ( $w r \breve{s}=j j w j b=j n m$ ' $w$ ) ist übrigens die genaue Umkehrung eines berühmten Verses aus dem Hohelied Salomonis "Ich schlief, aber mein Herz wachte" ( ${ }^{a} n \hat{i} j{ }^{e}$ šenāh $w^{e} l i b b \hat{i}$ 'èr Hhld 5,2). Gemeinsam ist beiden Texten der Gedanke einer Dissoziation von Herz und Selbst, dem redenden "Ich“. Dieser Gedanke der Dissoziation ist im ägyptischen Text ausgedrückt sowohl durch das Motiv des Schlafens und der Mattigkeit, als auch durch das der räumlichen Entfernung: „mein Herz ist nicht in meinem Leib“. Dieselbe Wendung kommt in der Geschichte des Sinuhe vor. Dort beschreibt sie einen Zustand extremer Furcht, der Sinuhe in Gegenwart Pharaos überkommt:

Während ich ausgestreckt auf meinem Bauch lag,

wußte ich nichts mehr von mir vor ihm, während dieser Gott mich freundlich grüßte.

Ich war wie ein Mann, der in der Finsternis gepackt wird.

Mein $\mathrm{Ba}$ war gegangen, meine Glieder zitterten, mein Herz war nicht mehr in meinem Leib, so daß ich Tod und Leben hätte unterscheiden können. ${ }^{22}$

Aber auch die Liebeskrankheit wird als Dissoziation von Herz und Selbst und dadurch ausgelöster Zerfall personaler Einheit beschrieben:

Mein Herz hüpft eilends davon, wenn ich an deine Liebe zu mir denke.

Es läßt mich nicht wie ein Mensch gehen und hüpft auf seinem Platze.

Nicht läßt es mich ein Kleid ergreifen.

Nicht nehme ich meinen Umhang.

Nicht lege ich Schminke an mein Auge.

Nicht salbe ich mich mit Wohlgeruch.

„Halt nicht an! Du erreichst das Ziel!“"

sagt es mir, so oft ich an ihn denke.

Mach mir, mein Herz, keinen Kummer!

Warum handelst du so töricht?

Warte gefaßt! Der Bruder kommt zu dir, dann will ich nach deinem Willen handeln.

$\mathrm{Laß}$ nicht die Menschen über mich sagen:

Jene Frau ist in Liebe gefallen!

Mögest du fest bleiben, so oft du an ihn denkst, mein Herz, und nicht fortspringen! $!^{23}$

pAnastasi IV, 4.11-5.5; Gardiner, Miscellanies, 39; Caminos, Miscellanies, 150 152; Schott, Liebeslieder, 116 Nr.57; Assmann, ÄHG Nr.184. 
Das Herz ist also zugleich das wichtigste und das problematischste Organ des Körpers. Es ist das Wichtigste, weil es das Zentrum der Person bildet und für Kohärenz, Bewußtsein, Identität, Verantwortlichkeit und Zurechnungsfähigkeit sorgt, und es ist das Problematischste aufgrund seiner Unstetigkeit, seiner Neigung zur Unruhe, zum Davonlaufen unter dem Einfluß starker Emotionen und Leidenschaften wie Furcht, Schrecken, Liebesverlangen, Sehnsucht und Heimweh. Kraft seines Herzens ist der ägyptische Mensch eine integrierte „Person“ und nicht einfach eine aggregierte oder additive Vielheit, wie dies von E. Brunner-Traut vertreten wurde $^{24}$ und wie es allenfalls vielleicht dem homerischen Menschenbild entspricht, das noch immer viel zu pauschal für das archaische Menschenbild schlechthin angesehen wird. Gerade durch seinen starken Begriff des Herzens als einer die personale Vielheit organisierenden Mitte unterscheidet sich das Alte Ägypten von vielen anderen Kulturen, steht aber gerade darin dem alttestamentlichen Menschenbild nahe. ${ }^{25}$ Aber das Herz wird zugleich verstanden als der Ort äußersten Ausgesetztseins gegenüber Einflüssen von außen und Trieben von innen.

Das Bild des leitenden Herzen und der Begriff des herz-geleiteten Menschen bezieht sich aber vor allem auf die soziale Dimension. Die Lehre vom Herzen, wie sie in den Grabinschriften und Lebenslehren seit dem Mittleren Reich ( $a b 2000$ v.Chr.) entwickelt wird, hat in ihrer reifen Form eine ausgeprägt anti-individualistische Tendenz. Großes Gewicht wird gelegt auf Einfügung, Unterordnung, „Schweigen“, Selbstkontrolle, Gehorsam, Altruismus, Zuverlässigkeit, Barmherzigkeit, Wohltätigkeit, Fairneß. Die schlimmsten Übel sind Egoismus, Habgier, Selbstdurchsetzung, Unabhängigkeit, Gewalttätigkeit, Aggressivität, Rücksichtslosigkeit, Leidenschaft, unkontrollierte Gefühlsausbrüche, hemmungsloses Sich-Gehenlassen. Das scheint eindeutig eine Reaktion gegen den selbstherrlichen Individualismus der Ersten Zwischenzeit (ca. 2150-2000), den Typus des $s h m-j b$,von mächtigem Herzen “26. Jetzt entsteht ein neuer Typus biographischer Selbstdarstellung, der ganz auf innere Fähigkeiten und Tugenden abgestellt ist. Ein typisches Beispiel findet sich auf einer der Stelen des Kammerherrn Antef, Sohnes der Senet, von der ich einige Zeilen anführen möchte:

24 Brunner-Traut, Frühformen des Erkennens.

25 Böhlig, Herz, hat gezeigt, daß hebräisches leb, lebāb in der LXX mit ver-

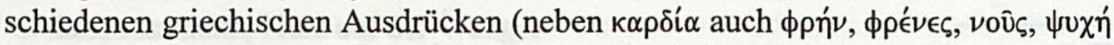
usw.) wiedergegeben wurde, während das Koptische wieder einheitlich auf hēt „Herz" zurückgreift.

Fecht, Cruces, 136f. 
Einzigartig geschickt, hervorragend an Rat, der sich auf die Worte derer stützt, die ihren Spruch kennen (wissen, was sie zu sagen haben), der ausgesandt wird wegen der Einschätzung seiner Geschicklichkeit, der dem Richter Bericht erstattet, der das Anliegen des Herzens kennt. Gepriesen von seinen Vorgesetzten, bekannt im Hause des Herrn, dessen Herz seine Geschäfte leitet, der seinen Arm beugt vor seinen Vorgesetzten, der geliebt wird von den Hofleuten des Königs.

Ein wohlbezeugter Name als Weiser, der ohne Schwanken dem Weg folgt, der das Wort hört in der Kapelle des Geb, eingeweiht in die Geheimnisse der Gerichtshalle. ${ }^{27}$

Die expliziteste Ausführung jedoch findet dieses Konzept des Herzens als des Sitzes innerer Qualitäten und als Führer der Person in der Inschrift einer Stele, die ein anderer Antef vier- oder fünfhundert Jahre später unter Thutmosis III. errichten ließ. Nicht nur mit seinem Namen, sondern auch mit seiner Autobiographie folgte er eng dem Vorbild des Mittleren Reichs:

Mein Herz war es, das mich antrieb, das zu tun entsprechend seiner Anleitung an mich.

Es legt für mich ein ausgezeichnetes Zeugnis ab, seine Anweisungen habe ich nicht verletzt.

Weil ich fürchtete, seine Anweisungen zu verletzen, gedieh ich dadurch über die Maßen.

Trefflich erging es mir wegen seiner Eingebungen für mein Handeln, tadelsfrei war ich durch seine Führung.

(...) Ein Gottesspruch ist es in jedem Leibe.

Selig der, den es auf den richtigen Weg des Handelns geführt hat! $!^{28}$

Mit der Vorstellung des Herzens als Sitz von Verantwortung und Gewissen hängt auch die Idee des Totengerichts zusammen, die sich in Ägypten gleichzeitig mit der Lehre des Herzens im Übergang vom Alten zum Mittleren Reich ausbildet und die für das ägyptische Menschenbild von entscheidender Bedeutung ist. Nach dieser Idee hat sich jeder Mensch nach seinem Tode vor einem jenseitigen Gerichtshof für seine Lebensführung zu verantworten, womit nicht nur Angst und Schrecken, sondern vor allem die enorme Verheißung verbunden ist, im Falle seiner Rechtfertigung aus dem Todeszustand erlöst und als „verklärter Ahnengeist" in die Unsterblichkeit der Götterwelt aufgenommen zu werden. Bei diesem Pro-

27 Lichtheim, Autobiographies, 107.

28 Louvre C 26: Urkunden IV 974f, s. Assmann, Sepulkrale Selbstthematisierung, $225 f$. 
zeß muß sich der Tote von einer langen Liste möglicher Verfehlungen lossagen, wobei sein Herz gegen eine Feder, das Symbol der Ma'at, gewogen wird. Jede Lüge würde die Waagschale des Herzens sinken lassen. Das Herz vertritt also den Toten im Sinne einer moralischen, für seine Taten verantwortlichen Person. ${ }^{29}$

\section{Konnektive Tugenden, diskonnektive Laster}

Das Herz ist also nach ägyptischer Auffassung das Prinzip sozialer und leiblicher Konnektivität. Hier treffen beide Leitungen zusammen. Es ist das Organ, das einen dazu befähigt, sich vom anderen leiten zu lassen und sich selbst zu koordinieren. Wenn das Herz diese Funktion nicht erfüllt, haben wir es mit pathologischen Fällen zu tun. Die Ausfälle auf leiblicher Ebene haben wir oben behandelt und wollen uns jetzt den Formen zuwenden, in denen das Herz auf sozialer Ebene versagt. In ägyptischen Augen ist das vor allem die Pathologie des Egoismus, die auf Torheit und Habgier zurückgeführt wird. Die alten Ägypter hielten solche Fälle für aussichtslos. Sie glaubten zwar, selbst den physischen Tod behandeln zu können. Vor Torheit und Habgier aber streckten sie die Waffen.

Die Erziehung zu konnektiven Tugenden ist das Thema der ägyptischen Lebenslehren. ${ }^{30}$ Sie behandeln in der Form der Kasuistik die Konnektivität der Lebenswelt, die der einzelne in seinem Tun und Verhalten fördern und bestärken, aber auch schädigen, gefährden und zerstören kann. Besonders aufschlußreich für die Vorstellung „diskonnektiver“ Haltungen und Verhaltensweisen ist ein Werk des Mittleren Reichs, das unter dem Titel „Die Klagen des Oasenmannes“ bekannt ist. Es könnte ebenso gut den Titel „Abhandlung über die Ma'at" tragen. ${ }^{31}$ Der Plot der Geschichte ist kurzgefaßt folgender: Ein Oasenmann, Repräsentant also nicht nur der sozialen Unterschicht, sondern auch noch der geographischen und kulturellen Peripherie des ägyptischen Reiches, ist seiner bescheidenen Habe beraubt worden und wendet sich an den zuständigen Magnaten und Großgrundbesitzer dieser Gegend, den Oberhofmeister Rensi, dem auch die Rechtspflege in diesem Gebiet obliegt. Der Oasenmann bringt seine Klage in so gewählten Worten vor, daß der Hofmeister den König von diesem ungewöhnlichen Auftritt unterrichtet. Der gibt die Anweisung, den beredten Kläger möglichst lange hinzuhalten, um ihm

S. dazu Assmann, Herz auf der Waage.

30 Eine handliche und lesbare Anthologie der wichtigsten ägyptischen Lebenslehren in deutscher Übersetzung besorgte Brunner, Altägyptische Weisheit. 
auf diese Weise noch weitere Reden von so wunderbarer Schönheit zu entlocken. Während der König unauffällig die Versorgung des Oasenmanns und seiner Familie sicherstellt, wird dieser weisungsgemäß von Rensi hingehalten. Der Kläger muß naturgemäß die Nichtbehandlung seines Falles falsch auslegen. Da der Beamte zum Schweigen verpflichtet ist, drehen sich alle Vorwürfe um den Tatbestand schuldhafter Nichtintervention. Wer an verantwortlicher Stelle gegen das Unrecht nicht einschreitet, wird an ihm mitschuldig.

Die neun Klagen gipfeln in dem orakelhaften Ausspruch:

Es gibt kein Gestern für den Trägen,

es gibt keinen Freund für den, der für die Ma'at taub ist,

es gibt kein Fest für den Habgierigen. ${ }^{32}$

Trägheit, Taubheit und Habgier werden hier als diskonnektive Haltungen herausgestellt. Er handelt nicht, wo gehandelt werden muß, weil ihm das „Gestern“, das soziale Gedächtnis fehlt. Er hört nicht auf das, was ihm vorgehalten wird: er ist unfähig zum Hören, das die Grundlage jeder Erkenntnis und jeder gerechten Entscheidung bildet, und ist damit auch unfähig zur „Freundschaft“, dem lebenspendenden Prinzip, sich vom anderen leiten zu lassen. Er kennt drittens kein „Fest“, keine gesellige Verausgabung, weil er in seiner Selbstsucht gleichsam eingemauert ist. Zum Thema der Taubheit, des Nicht-Hörens auf die Worte der Ma'at äußert sich vor allem die Lehre des Ptahhotep, in der das Hören eine geradezu leitmotivische Rolle spielt. Diese Lehre ist uns in einer Reihe von Papyri und Ostraka überliefert, gehörte also zu den großen, in der Schule auswendig zu lernenden „kulturellen Texten“ und stammt vermutlich aus dem frühen Mittleren Reich (bald nach 2000):

Der Tor $^{33}$ aber, der nicht hört,

für den wird nichts getan;

Wissen sieht er als Unwissen an,

Förderliches als Schädliches:

Alles Schändliche tut er,

so daß Klage geführt wird über ihn Tag für Tag.

Er lebt von dem, woran man stirbt, seine verderbliche Nahrung ist Sprechen.

Seine Verfassung darin ist den Fürsten bekannt, nämlich: lebendig tot zu sein Tag für Tag.

33 Wörtl. „der Suchende“, s. Seibert, Charakteristik, 78. Junge, Lehre Ptahhoteps, übersetzt „der Unwissende“. Es handelt sich aber nicht nur um Unwissenheit, sondern um konstitutive Unbelehrbarkeit. 
Man geht vorüber an seinen Notlagen

wegen der Menge des ihm Widerfahrenden, Tag für Tag. ${ }^{34}$

Hier wird Verstocktheit oder Torheit geradezu als sozialer Tod bezeichnet. So wie einer lebt, wenn der andere ihn geleitet, so stirbt einer, wenn er zu solchem Geleit - d.h. zur Konnektivität und Konvivenz - unfähig ist. Wer unfähig ist zu verstehen, kann zwischen Gut und Böse nicht unterscheiden. Schädliches sieht er als förderlich, Förderliches als schädlich an. So ist er nicht nur unfähig, anderen etwas Gutes zu tun, sondern es kann auch ihm selbst nichts Gutes getan werden. Seine soziale Inkompetenz isoliert ihn aus dem Gefüge des Füreinanderhandelns und Einanderleitens. Zum Leben gehört Aufgeschlossenheit für die leitenden Impulse des anderen. Solche Aufgeschlossenheit nennt der Ägypter „Hören“ “35 Das Ideal ist das „hörende Herz“ ${ }^{36}$ Die Konstellationen der Konnektivität werden vor allem vermittelst der Sprache geknüpft. Wer nicht auf die anderen hören kann, ist zur Einsamkeit und damit zum Tode verurteilt.

Auch zum Thema Habgier nimmt der weise Ptahhotep Stellung:

Wenn du willst, daß deine Führung vollkommen sei,

dann halte dich fern von allem Bösen

und sei gewappnet gegen ein Vorkommnis von Habgier.

Sie ist eine schwere, unheilbare Krankheit,

die man nicht behandeln kann.

Sie entfremdet Väter und Mütter

samt den Vollbrüdern;

sie vertreibt die Gattin.

Ein Erwählen ist sie von allem Schlechten, ein Behältnis ist sie von allem Verwerflichen.

Fortdauert (hingegen) der Mann, der der Ma'at entspricht und der fortgeht (stirbt) entsprechend seinem Gang.

Er ist es, der dadurch ein Testament machen kann.

Aber der Habgierige hat kein Grab. ${ }^{37}$

Wie Verstocktheit als Tod, so wird die Habgier als unheilbare Krankheit diagnostiziert. Sie gilt als das Prinzip der sozialen Desintegration, als Inbegriff diskonnektiven (unsolidarischen, Gemeinschaft zerstörenden)

34 Ptahhotep 575ff, pPrisse 17, 4-9; Zaba, Maximes, 60f; Junge, Lehre Ptahhoteps, 89f. Meine Übersetzung folgt weitgehend der meisterhaften Bearbeitung der Stelle durch Seibert, Charakteristik, 78ff.

35 Zur Bedeutung des Hörens in der Einschätzung der Ägypter s. Assmann, Ma'at, $73 \mathrm{ff}$.

S. dazu Brunner, Das hörende Herz.

37 Ptahhotep 298-315; Brunner, Altägyptische Weisheit, 119f Verse 237-251; Junge, Lehre Ptahhoteps, $50 \mathrm{f}$. 
Verhaltens. ${ }^{38}$ Sie zerreißt die allerengsten Bindungen der Blutsverwandtschaft und der Ehe und wirkt auf diese Weise isolierend, vereinsamend in der Sozialdimension. Das Unheil solcher Vereinsamung erweist sich aber beim Sterben: der Habgierige hat kein , Grab', d.h. keinen Ort im sozialen Gedächtnis, das den Tod überdauert. Das ist ja das Geheimnis der Konnektivität in den Augen der Ägypter: sie überdauert den Tod und verheißt Unsterblichkeit. Verstocktheit und Habgier vermögen dieses Netz zu zerreißen, aber nicht der Tod. Das ist die Hoffnung, die die Grundlage der ägyptischen Kultur bildet. Das ägyptische Ideal der Ma'at ist nicht nur das Prinzip der sozialen Konnektivität, sondern auch der Dauer, des Bestandes und damit der zeitlichen Konnektivität, der Erinnerung, der Kontinuität von Vergangenheit und Zukunft. Der Egoist hat nicht nur keine Freunde, er hat auch keine Zukunft. Er hat keine Aussicht auf anamnetische Solidarität, und damit keine Aussicht auf ein Grab. Denn ohne solche Aufgehobenheit im sozialen Gedächtnis hat das materielle Grab keinen Sinn, es ist ein deutungsloses Zeichen, das der Vergänglichkeit preisgegeben ist. Habgier zerstört die sozialen Bindungen (In den Klagen des Oasenmannes heißt es lapidar: „Habgier zerstört die Freundschaft ${ }^{(39}$ ), und sie zerstört - in der Zeitdimension - die Dauer, die Einbindung des einzelnen in die Fortdauer der Gruppe und ihrer Erinnerung. Das Schicksal des Egoisten ist Einsamkeit, und Vergessen, gedächtnisloses Verschwinden ist das Schicksal des Einsamen:

Das Denkmal eines Mannes ist seine Tugend, der mit schlechtem Charakter aber wird vergessen. ${ }^{40}$

In der Lehre des Ptahhotep heißt es:

Der gute Charakter wird zu einem, dessen gedacht wird. ${ }^{41}$

Damit kommen wir zur dritten Form diskonnektiven Verhaltens, zum "Trägen, der kein Gestern (d.h. kein Gedächtnis) hat". Nach ägyptischer Auffassung ist das Gedächtnis der Sozialsinn par excellence. Dazu wieder die Klagen des Oasenmannes. „Ein guter Charakter kehrt zurück an seine Stelle von gestern, denn es ist befohlen: Handle für den, der handelt, um zu veranlassen, daß er tätig bleibt. Das heißt, ihm danken für das, was er getan hat““ ${ }^{42}$ Nur der kann tätig teilnehmen an der sozialen Konnektivität,

$38 \mathrm{Vgl}$. dazu die eingehende Analyse von Seibert, Charakteristik, $78 \mathrm{ff}$.

39 B1, 170, s. Assmann, Ma'at, 87.

40 Stele London UC 14333 ed. Goedicke, Wisdom Text, 26, vgl. Schenkel, Weisheitslehre, $11 \mathrm{f}$.

41 Ptahhotep 494, nach Junge, Lehre Ptahhoteps, 92.

42 Die Klagen des Oasenmannes (Bauer) B 1, 109-110; Vogelsang, Kommentar, 100. 
der „an seine Stelle von gestern“ zurückkehren, d.h. sich erinnernd auf die Vergangenheit beziehen kann. Umgekehrt liest man in einem anderen Text ${ }^{43}$ dieser Zeit: „Man erinnert sich nicht des Gestern, man handelt nicht für den, der gehandelt hat heutzutage. ${ }^{\text {"44 }}$ Der Verlust der Vergangenheit ist gleichbedeutend mit dem Zerfall aller sozialen Bindungen. Wenn das soziale Gedächtnis zerfällt, ägyptisch gesprochen „das Gestern vergessen wird“, wird die Welt, wie es ein weiterer Text ausdrückt, zum Kampfplatz eines Kampfes aller gegen alle: „Siehe, man kämpft auf dem Kampfplatz, denn das Gestern ist vergessen. Nichts gelingt dem, der den nicht mehr kennt, den er gekannt hat. “45

Das Gedächtnis, so läßt sich folgern, gehört nicht zum Egoismus, zur Selbsterhaltung und Triebbefriedigung, sondern zum Altruismus, zum Denken an andere und ans Ganze der Gruppe, der Gemeinschaft, der Gesellschaft, der Menschheit. Ein Gedächtnis braucht der Mensch, nach altägyptischer Vorstellung, um sich einzufügen und dazuzugehören. Das Gedächtnis macht ihn zum Mitmenschen, befähigt ihn zu einem Leben in Gemeinschaft. Das trifft sich auf überraschende Weise mit Nietzsches Überlegungen zur Genealogie der Moral. Für Nietzsche entwickeln sich Moral und Gedächtnis koevolutiv im Prozeß der Züchtung des Menschen zum Mitmenschen, d.h. Kulturmenschen. ${ }^{46}$ Gesellschaft beruht darauf, daß sich die Menschen „ein Gedächtnis machen“. Nietzsche entwickelt diesen Begriff am Paradigma der im Hinblick auf zukünftige Leistungen eingegangenen Verpflichtung und definiert den gedächtnisfähigen Menschen als das "Tier, das versprechen darf" 47

43 pBerlin 3024, 115f, hg. Erman, Gespräch. Zahlreiche neue Übersetzungen, u.a. von Hornung, Gesänge, 115.

44 Diese drei Zeilen stehen im Text an herausgehobener Stelle. Sie bilden die mittlere Strophe eines ,anaphorischen Strophenliedes“, dessen Strophen alle mit dem Satz „Zu wem kann ich heute (noch) reden?" beginnen und das mit drei anderen Strophenliedern den ansonsten ,prosaischen“ (oder besser gesagt: anders und schwächer geformten) Text lyrisch unterbricht.

45 Lehre des Königs Amenemhet I, pMillingen 10f; Abschnitt V d-e in der Textausgabe von Helck, Amenemhet, 35ff.

46 S. insbesondere die Schrift ,Zur Genealogie der Moral“, Zweite Abhandlung.

47 Nietzsche, Werke II, 799f: „Eben dieses notwendig vergeßliche Tier, an dem das Vergessen eine Kraft, eine Form der starken Gesundheit darstellt, hat sich nun ein Gegenvermögen angezüchtet, ein Gedächtnis, mit Hilfe dessen für gewisse Fälle die Vergeßlichkeit ausgehängt wird - für die Fälle nämlich, daß versprochen werden soll: somit keineswegs bloß als ein passivisches Nicht/wieder/los/ werden/können des einmal eingeritzten Eindrucks, ... sondern ein aktives Nichtwieder-los-werden-wollen, ein fort-und-fort-wollen des einmal Gewollten, ein eigentliches Gedächtnis des Willens.“ Das, sagt Nietzsche, ist „die lange Geschichte von der Herkunft der Verantwortlichkeit". 


\section{Polytheismus als konstellative Theologie}

Wie leicht zu sehen, entspricht dieser konstellative Personbegriff vollkommen der Struktur einer polytheistischen Religion. Auf diese Zusammenhänge kann ich hier nicht eingehen, aber möchte aus systematischen Gründen doch wenigstens im Vorübergehen kurz darauf verweisen. ${ }^{48}$ Die alten Ägypter assoziierten die Einheit und Einsamkeit Gottes mit dem Chaos, dem Zustand der Welt vor (und nach) ihrer Existenz, die nicht als Schöpfung aus dem Nichts, sondern Entfaltung aus der Eins gedacht wird. In der Wirklichkeit ist das Göttliche nur „konstellativ“ denkbar, von einer Gottheit kann nur in Bezug auf andere Gottheiten geredet werden, mit denen sie in den Rollen mythischer Handlungszusammenhänge verbunden ist. Wenn das Göttliche in personaler Form konzipiert wird, dann muß diese Form notwendigerweise eine „konstellative“ Struktur aufweisen. Daher vermögen neben Totenliturgien und Grabinschriften auch die Götterhymnen den konstellativen Personbegriff der Ägypter zu veranschaulichen. Sie schildern und preisen die Götter als Personen, und das heißt: in wechselseitigen Beziehungen des Miteinander-Handelns und Miteinander-Sprechens. Was die Götter als Personen sind, sind sie nur in Bezug aufeinander. Dadurch, daß sie in festen Konstellationen ihre bestimmte Rolle spielen, konstituieren und konturieren sich wechselseitig ihre Personalitäten. Konstellative Theologie und Anthropologie betonen die Bindungen, Rollen und Funktionen, durch die die konstituierenden Glieder miteinander verknüpft werden. Was sie als schlimmstes Übel ansehen, sind die Vorstellungen der Isolierung, Einsamkeit, Selbstgenügsamkeit und Unabhängigkeit. In ihrer Sicht sind das Symptome von Tod, Auflösung und Zerstörung. Leben heißt Interdependenz, Kommunikation, Einbezogenheit in die Beziehungsnetze, aus denen die Wirklichkeit besteht. Die Fähigkeit zur Einsamkeit haben die Menschen vermutlich erst im Zeichen des Monotheismus gelernt. In diesem Zusammenhang ist es erstaunlich, daß die Ägypter, die im Rahmen ihrer traditionellen Religion und ihrer konstellativen Anthropologie die soziale Natur des Menschen so stark betonten, mit der Annahme des christlichen Monotheismus ins genaue Gegenteil umschlugen und mit dem heiligen Antonius und seinen zahllosen Nachfolgern, den "Wüstenvätern“ zu den Pionieren radikaler Weltflucht und Einsamkeitssuche geworden sind. ${ }^{49}$

48 Diese Beziehungen zwischen Personbegriff und Polytheismus habe ich in meiner Dissertation „Liturgische Lieder" aufzuhellen versucht und im IV. Kapitel meines Buchs „Ägypten“, 102ff zusammenfassend dargestellt.

49 Vgl. hierzu Assmann / Assmann (Hg.), Einsamkeit, und darin besonders den Beitrag von Barasch. 


\section{Staatskunst und Gemeinschaftskunst ${ }^{50}$}

Daß ein „konstellativer“ Personbegriff und eine darauf aufbauende hochelaborierte Gemeinschaftskunst sehr gut zu einer Gesellschaft passen, die den ersten großräumigen Staat, von dem die Geschichte weiß, errichtet und über mehr als drei Jahrtausende perpetuiert hat, leuchtet ein. Ein solches Projekt war nur auf der Grundlage starker konnektiver Tugenden, Ideale und Normen zu errichten und aufrecht zu erhalten. Auch auf diesen Zusammenhang, den wir hier nicht entfalten können, soll abschließend wenigstens kurz verwiesen werden. Die ägyptische Gemeinschaftskunst ist eine Beziehungsethik, im Unterschied zur abendländischen, auf Aristoteles zurückgehenden Tradition einer individuellen Glücks- oder Strebensethik. Die alten Ägypter entwickelten die Moral aus den Beziehungen heraus, die die Menschen zur Gemeinschaft verbinden.

Ma'at stellt nicht nur Gemeinschaft her zwischen den Göttern, den Menschen und den Toten, sondern auch zwischen den Ereignissen. Auf der Ebene der Ereignisse entspricht $\mathrm{Ma}^{\prime}$ at daher dem, was man auch „Sinn“ nennen könnte, denn Sinn läßt sich in erster Linie als „Zusammenhang" oder „Konnektivität" der Handlungen und Ereignisse definieren. Wir machen die Erfahrung der Sinnlosigkeit vor allem, wenn wir nicht sehen, wie eines mit dem anderen zusammenhängt. Im alten Orient, vielleicht überhaupt in der ganzen alten Welt, erblickte man den Sinn des Geschehens darin, daß „die Tat zum Täter zurückkehrt". ${ }^{51}$ Nichts bleibt folgenlos, irgendwann und irgendwie rächt sich das Böse und lohnt sich das Gute. Dies Prinzip nennt man den Tun-Ergehen-Zusammenhang. Nach ägyptischer Auffassung sind die Menschen für diesen Zusammenhang selbst verantwortlich. Sie stellen ihn her, sie halten ihn in Gang. Das ist weder die Sache eines göttlichen Willens noch einer immanenten Weltordnung. ${ }^{52}$

Die Menschen haben zwei Möglichkeiten, die Ma'at zu verfehlen, als einzelne und als Gesellschaft. Wenn der einzelne sich nicht an die Ma'at hält, dann scheitert er, wenn nicht zu Lebzeiten, dann im Tod. Für ihn bedeutet der Tod dann das Ende, über das ihn keine Kontinuität hinwegrettet. Wenn die Gesellschaft die Ma'at verfehlt, dann lehnt sie sich gegen den Staat auf und zerstört das Königtum. Damit verschwinden die Rahmenbedingungen dafür, daß der einzelne sich an die Ma'at halten und seinem Dasein über den Tod hinaus Bestand verleihen kann. Dann ver-

S. hierzu auch Assmann, Ma'at - Gemeinschaftskunst, 37ff.

Janowski, Tat, $167 \mathrm{ff}$.

52 Erst in der Spätzeit, also im 1.Jt. v.Chr. wird der Zusammenhang von Tun und Ergehen dem Willen Gottes anheimgestellt, s. dazu Assmann, Ma'at, 66ff. 
schwindet der Sinn aus der Welt, und die Welt geht aus den Fugen. Unter den Menschen herrschen Mord und Totschlag, die Stärkeren erschlagen die Schwächeren, Recht und Ordnung, Sicherheit und Vertrauen verschwinden aus der Welt, und das Band zwischen Menschen und Göttern zerreißt. Das wird in der literarischen Gattung der Klagen ausführlich ausgemalt.

In den Klagen des Oasenmannes wird staatliche Autorität von unten eingeklagt, weil sie - scheinbar - von oben nicht ausgeübt wird. Die Pointe der Geschichte ist, daß hier ein Mensch aus der untersten Schicht und der äußersten Peripherie des Reiches die Herrschenden an ihre Pflicht erinnert, ihre Macht auszuüben, um die Ma'at auf Erden durchzusetzen. Der Staat ist um der Kleinen und Schwachen willen da. So wie Ma'at die Bedingungen dafür schafft, daß die Menschen sich zur Gemeinschaft verbinden, so schafft der Staat die Grundlage dafür, daß auf Erden Ma'at herrscht und nicht deren Gegenteil, Isfet. Daher lautet die erste Lehre der Gemeinschaftskunst, daß es auf Erden einen Staat geben muß. Zum Tun und Sagen der Ma'at, zur Herstellung von Sinn und Zusammenhang, sind die Menschen aus eigener Kraft nicht in der Lage; sie bedürfen dazu des Staates, der vom Schöpfergott selbst auf Erden eingesetzt ist, um hier die Ma'at zu verwirklichen.

Das bringt ein sehr zentraler, oft kopierter Text von offenbar kanonischem, d.h. im höchsten Maße normativem Rang zum Ausdruck, der in den königlichen Sonnenkultstätten des Neuen Reichs aufgezeichnet wurde. Es handelt sich um einen „kulttheologischen Traktat“ über den König als Anbeter des Sonnengottes:

$\mathrm{RE}$ hat den König NN eingesetzt

auf der Erde der Lebenden

für unendliche Zeit und unwandelbare Dauer

beim Rechtsprechen den Menschen, beim Zufriedenstellen der Götter, beim Verwirklichen der Wahrheit, beim Vernichten der Sünde ( $j s f t$; er gibt den Göttern Opferspeisen, und den Verklärten Totenopfer. ${ }^{53}$

Der König verwirklicht die Ma'at durch Recht und Kult: indem er den Menschen Recht spricht und den Göttern und Toten Opfer darbringt. In allen Sphären stellt die Verwirklichung der Ma'at Zusammenhang und Gemeinschaft her. Der Kult sorgt für den Einklang von Himmel, Erde und Totenreich, und das Recht sorgt für Gemeinschaft und Ordnung unter den Menschen. 
Als Gerechtigkeit ist Ma'at nicht die blinde iustitia der römischen Tradition, die ohne Ansehen der Person Recht spricht, sondern die „rettende Gerechtigkeit" der orientalischen Tradition, die ihre Urteile in hellsichtigster Erkenntnis der unter den Menschen herrschenden Ungleichheit fällt und ihre Hauptaufgabe, den Inbegriff gerechter Rechtsprechung, darin sieht, die Schwachen vor den Starken zu schützen. ${ }^{54}$ Gerechtigkeit ist dieser Tradition zufolge nicht die blinde Vollstreckung des Gesetzes, sondern eine Rechtsprechung in Ansehung der Person, die geltendes Recht im Einzelfall auch einmal außer Kraft setzen kann, wenn es zu unmenschlichen Härten führte, und mit Gnade ebensoviel zu tun hat wie mit Recht. Nach altorientalischer und biblischer Rechtsauffassung dient das Gesetz nicht vornehmlich dem Schutz des Eigentums; im Gegenteil spielt der Gedanke gesetzlich vorgeschriebenen Verzichts auf Eigentum, etwa in der Form von Schulderlassen, eine große Rolle. Typische Verzichtgesetze sind etwa Edikte der Begnadigung, Freilassung, Amnestie, wie sie mesopotamische und ptolemäische Herrscher anläßlich ihrer Thronbesteigung zu erlassen pflegten - also ein Akt, den wir eher als Rechtsaufhebung denn als Rechtsstiftung einstufen würden.

„Richten“ bedeutet in erster Linie „schlichten“. Dabei geht es nicht um die Vollstreckung von Gesetzen einer zeitenthobenen Gerechtigkeit, sondern um die Wiederherstellung von Harmonie und Gemeinschaft durch die Schlichtung von Streit und Zwietracht. Das Ideal des guten Richters ist Thot, der Gott, „der die streitenden Brüder (Horus und Seth) trennte, so daß beide zufrieden waren". Der letzte Zweck des Urteils besteht nicht in der Bestrafung des Übeltäters, sondern in der Wiederherstellung der gestörten Harmonie. Ohne den Staat würde also nach ägyptischer Auffassung nicht Ma'at auf Erden herrschen, sondern Isfet.

Die kompakte Formel des Königstextes: „den Menschen Recht sprechen" wird im 126. Kapitel des Totenbuchs ersetzt durch das Prinzip der „rettenden Gerechtigkeit": „Recht sprechen zwischen dem Armen und dem Reichen (bzw. „dem Schwachen und dem Mächtigen“). Es handelt sich um eine Anrufung an „die vier Paviane am Bug der Sonnenbarke“,

die die Ma'at aufsteigen lassen zum Allherrn,

die Recht sprechen zwischen dem Schwachen und dem Starken, die die Götter befriedigen mit dem Hauch ihres Mundes, die den Göttern Gottesopfer und den Verklärten Totenopfer geben. ${ }^{55}$

\footnotetext{
54 S. dazu Janowski, Rettende Gerechtigkeit; Assmann / Janowski / Welker (Hg.), Gerechtigkeit. 
Damit wird klar, worin jene Isfet besteht, der der König durch Rechtsprechung abhelfen bzw. gegensteuern muß: in der Ungleichheit der Menschen. Gerichtet wird nicht zwischen dem Guten und dem Bösen - saddîq und rāšă ', wie es biblisch heißen würde -, sondern zwischen dem Armen und dem Reichen, dem Schwachen und dem Starken. Damit wird unterstellt, daß der Reiche oder Starke der Böse ist. Das heißt: das Böse liegt in der menschlichen Natur, die, wenn man sie nur gewähren läßt, alle Chancen von Macht, Reichtum, Stärke und Einfluß nutzen wird, um die Schwächeren zu unterdrücken. Nicht die Ungleichheit als solche ist das Böse, sondern die Unterdrückung der Schwachen durch die Starken, die damit unvermeidlich verbunden ist. ${ }^{56}$

Die Literaturgattung der „Klagen“ schildert den Zustand einer Welt ohne Staat. Sie haben die Funktion, einzuschärfen, daß nicht nur Frieden, Ordnung und Gerechtigkeit, sondern sogar der Sinn der Schöpfung und das natürliche Gedeihen von der Existenz des Staates abhängen. Wenn der Staat zerfällt oder wenn er, wie in den Klagen des Oasenmannes unterstellt, seine Macht nicht ausübt, dann verschwindet Ma'at aus der Welt. Alle Gemeinsamkeit zerfällt: Sprache, Wissen, Erinnerung. Wenn die Erinnerung schwindet, lohnt sich das Gute, rächt sich das Böse nicht mehr, die Zirkulation des Sinns bricht zusammen. Die Menschen verstehen sich nicht mehr, an die Stelle der Sprache tritt die Gewalt. Auch die Gemeinschaft mit den Göttern zerbricht. Die Götter wenden sich ab, die Natur verliert ihre nährenden Segenskräfte. Hungersnot und Verelendung sind die Folge. Dann herrschen unter den Menschen Mord und Totschlag. Alle Bindungen sind gelöst. Väter und Söhne bringen sich gegenseitig um. ,Wenn drei auf der Straße gehen, findet man nur noch zwei: denn die größere Zahl tötet die kleinere", wie es in einer anderen Klage, den Mahnworten des Ipuwer heißt. Der Nil färbt sich rot von Blut. Die Welt fällt zurück in den maatlosen Naturzustand der gegenseitigen Unterdrückung, des Faustrechts, der Gewalt.

Die Verwirklichung der Ma'at ist also die vornehmste Aufgabe des Staates. Sie entspringt klar dem Willen Gottes, aber Gott delegiert sie an den König. Er selbst greift nicht ein, um sie auf Erden durchzusetzen. Allerdings ist dieser Gott auch kein deus otiosus, der sich nach getaner Schöpfungsarbeit von den Menschen abwendet und im Himmel zur Ruhe setzt. Ganz im Gegenteil: als Sonnengott umkreist der Schöpfer unermüdlich die Erde und durchreist Himmel und Unterwelt, Götterwelt und Totenreich, um dort die Ma'at zu verwirklichen und die Isfet zu vertreiben. Auch auf dieser Ebene ist Ma'at also keine immanente Weltordnung, 
sondern eine immer wieder neu gegen Widerstände durchzusetzende Rechtsordnung. Immanent ist der Welt vielmehr eine Gravitation zu Chaos, Zerfall und Entropie, die sich in der Gestalt eines riesigen Wasserdrachens verkörpert und vom Sonnengott mit dem Beistand vieler anderer Götter unablässig bekämpft werden muß.

Was die Ägypter dafür tun, ist einerseits der unablässige Vollzug ungezählter Rituale, die die kosmischen Vorgänge auf Erden begleiten und dadurch die irdische Welt dem kosmischen Prozeß einfügen und anpassen, und andererseits eben der Staat, der die Ma'at-durchsetzende Herrschaft des Sonnengottes auf Erden abbildet. Wenn der Staat zerfällt und die Riten nicht mehr durchgeführt werden, verliert die Erde ihre Ähnlichkeit mit dem Himmel, die Analogie zerfällt, die Spiegelung trübt sich und der Sinn verschwindet. Wenn wir Ma'at und „Sinn“ gleichsetzen, dann muß nach ägyptischer Auffassung dieser Sinn unablässig hergestellt und der Welt in Form von Riten und Rechtsprechung unablässig gewissermaßen eingehaucht werden. Der König verwirklicht die Ma'at, so hatte es der oben zitierte Text ausgedrückt, indem er den Menschen Recht spricht und den Göttern und Toten Opfer darbringt, also durch Recht und Kult. Der Kult sorgt für den Einklang von Himmel, Erde und Totenreich, und das Recht sorgt für den Schutz der Schwachen vor den Starken.

\section{Literatur}

Assmann, A. / Assmann, J. (Hg.), Einsamkeit, München 2000

Assmann, J., Liturgische Lieder an den Sonnengott, Berlin 1969

—, Sepulkrale Selbstthematisierung im alten Ägypten, in: A. Hahn / V. Kapp (Hg.), Selbstthematisierung und Selbstzeugnis: Bekenntnis und Geständnis, Frankfurt 1987, 208-232

- Ma'at. Gerechtigkeit und Unsterblichkeit im Alten Ägypten, München 1990/ ${ }^{5} 2006$

-, Ägypten - Theologie und Frömmigkeit einer frühen Hochkultur, Stuttgart ${ }^{2} 1991$

-, Das Bild des Vaters im alten Ägypten, in: ders., Stein und Zeit. Mensch und Gesellschaft im Alten Ägypten, München 1991, 115-137

- Zur Geschichte des Herzens im alten Ägypten, in: ders. / Th. Sundermeier (Hg.), Die Erfindung. des inneren Menschen. Studien zur religiösen Anthropologie, Gütersloh 1993, 81-112

- , Ägyptische Hymnen und Gebete (OBO), Freiburg (Schweiz) / Göttingen ${ }^{2} 1999$

- Das Herz auf der Waage. Schuld und Sünde im Alten Ägypten, in: T. Schabert / D. Clemens (Hg.), Schuld, ErJb 7 (1999) 99-147 bzw. J. Assmann, Herrschaft 
und Heil. Politische Theologie in Altägypten, Israel und Europa, München 2000, 133-177

—, Tod und Jenseits im Alten Ägypten, München 2001

-, Die Verborgenheit des Mythos in Ägypten, in: ders., Ägyptische Geheimnisse, München 2004, 31-57

-, Altägyptische Totenliturgien II. Totensprüche und Liturgien in Gräbern des Neuen Reichs (Suppl. Schriften der Heidelberger Akademie der Wissenschaften), Heidelberg 2005

-, Ma'at - Gemeinschaftskunst im Alten Ägypten, in: ders. / E. Krippendorff / H. Schmidt-Glintzer, Ma'at, Konfuzius, Goethe. Drei Lehren für das richtige Leben, Frankfurt a.M. 2006, 23-69

- / Janowski, B. / Welker, M. (Hg.): Gerechtigkeit. Richten und Retten in der abendländischen Tradition und ihren altorientalischen Ursprüngen, München 1998

Barasch, M., The Hermit in the Desert: an Image of Solitude, in: A. Assmann / J. Assmann (Hg.), Einsamkeit, München 2000, 153-172

Blackman, A.M., Middle Egyptian Stories (BAeg II), Brüssel 1932

Böhlig, A., ,Herz' in der Übersetzung des koptischen Neuen Testaments, in: $M$. Görg (Hg.), Fontes atque pontes. Festgabe für Hellmut Brunner ( ̈AT 5), Wiesbaden $1983,47-61$

Borioni, G., Der Ka aus religionswissenschaftlicher Sicht (Veröffentlichungen der Institute für Afrikanistik und Ägyptologie der Universität Wien), Wien 2005

Brunner, H., Art. Herz, LÄ II (1977) 1158-1168

—, Altägyptische Weisheit. Lehren für das Leben, Zürich / München 1988

-, Das Herz im ägyptischen Glauben, in: Das Herz im Umkreis des Glaubens I, Biberach 1965, 81-106, wiederabgedr. in: ders., Das hörende Herz. Kleine Schriften zur Religions- und Geistesgeschichte Ägyptens (OBO 80), Freiburg (Schweiz) / Göttingen 1988, 8-44

- Das hörende Herz. Kleine Schriften zur Religions- und Geistesgeschichte Ägyptens (OBO 80), Freiburg (Schweiz) / Göttingen 1988

Brunner-Traut, E., Der menschliche Körper - eine Gliederpuppe, ZÄS 115 (1988) 814

—, Frühformen des Erkennens. Am Beispiel Altägyptens, Darmstadt ${ }^{2} 1992$

Burkard, G., Ptahhotep und das Alter, ZÄS 115 (1988) 19-30

Caminos, R.A., Late Egyptian Miscellanies, Oxford 1952

Crüsemann, F., Versuch über die drohende Vereinsamung Gottes, in: A. Assmann /J. Assmann (Hg.), Einsamkeit, München 2000, 113-127

Donadoni, S. (Hg.), Der Mensch im Alten Ägypten, Frankfurt a.M. / New York 1992 Erman, A., Das Gespräch eines Lebensmüden mit seiner Seele, Berlin 1896 
Fecht, G., Cruces Interpretum in der Lehre des Ptahhotep (Maximen 7, 9, 13, 14) und das Alter der Lehre, in: Hommages à François Daumas, Montpellier 1986, 227251

Freud, S., Totem und Tabu, Wien 1913

Gardiner, H.A., Late Egyptian Miscellanies (BAeg 7), Brüssel 1937

Goedicke, H., A New Wisdom Text: Stele London UC 14333, JEA 48 (1962) 26

Greven, L., Der Ka in Theologie und Königskult des Alten Reiches, Glückstadt 1952 Helck, W., Die Lehre des Amenemhet, Wiesbaden 1969

Hornung, E., Gesänge vom Nil, Zürich 1990

Janowski, B., Die Tat kehrt zum Täter zurück. Offene Fragen im Umkreis des Tun-

Ergehen-Zusammenhangs, in: ders., Die rettende Gerechtigkeit. Beiträge zur Theologie des Alten Testaments 2, Neukirchen-Vluyn 1999, 167-191

-, Die rettende Gerechtigkeit. Beiträge zur Theologie des Alten Testaments 2, Neukirchen-Vluyn 1999

Jansen-Winkeln, K., ,Horizont" und ,Verklärtheit': zur Bedeutung der Wurzel 'b, SAK 23 (1996) 201-215

Junge, F., Die Lehre Ptahhoteps und die Tugenden der ägyptischen Welt, Göttingen 2003

Junker, H., Die Götterlehre von Memphis (SPAW 23), Berlin 1940

-, Die politische Lehre von Memphis (SPAW 6), Berlin 1941

Kaiser, O. (Hg.), Texte aus der Umwelt des Alten Testaments II/6, Gütersloh 1991

Klasens, A., A Magical Statue Base (Socle Behague) in the Museum of Antiquities at

Leiden (Oudheidkundige Mededelingen uit het Rijksmuseum van Oudheden te Leiden N.R. XXXIII), Leiden 1952

Koch, R., Die Erzählung des Sinuhe (BAeg 17), Brüssel 1990

Kucharek, A., Die Klagelieder der Isis und Nephthys in Texten der griechisch-römischen Zeit, Diss. Heidelberg 2003

Kusber, E., Der altägyptische Ka - „Seele“ oder „Persönlichkeit“?, Tübingen 1994

Lichtheim, M., Ancient Egyptian Autobiographies Chiefly of the Middle Kingdom. A

Study and an Anthology (OBO 84), Freiburg (Schweiz) / Göttingen 1988

Nietzsche, F., Zur Genealogie der Moral, in: Werke in drei Bänden, hg. von K.

Schlechta, Bd.2, München 1960, 761-900

Parkinson, R.B. (ed.), The Tale of the Eloquent Peasant, Oxford 1991

- The Tale of Sinuhe and Other Ancient Egyptian Poems, Oxford 1997

Quack, J.F., Die Lehren des Ani. Ein neuägyptischer Weisheitstext in seinem kulturellen Umfeld (OBO 141), Freiburg (Schweiz) / Göttingen 1995

Sander-Hansen, C.E., Die Texte der Metternichstele (AAeg VII), Kopenhagen 1956

Schenkel, W., Eine neue Weisheitslehre?, JEA 50 (1964) 6-12

- Soziale Gleichheit und soziale Ungleichheit und die ägyptische Religion, in: $G$. Kehrer (Hg.), „Vor Gott sind alle gleich“. Soziale Gleichheit, soziale Ungleichheit und die Religionen, Düsseldorf 1983, 26-41 
Schott, S., Altägyptische Liebeslieder, Zürich 1950

Schweitzer, U., Das Wesen des Ka im Diesseits und Jenseits der Alten Ägypter (ÄF 19), Glückstadt 1956

Seibert, P., Die Charakteristik. Eine ägyptische Sprechsitte und ihre Ausprägungen in Folklore und Literatur, Wiesbaden 1967

Sethe, K., Die altägyptischen Pyramidentexte, Leipzig 1908-1922

—, Dramatische Texte zu altägyptischen Mysterienspielen, Leipzig 1928

-, Urkunden des ägyptischen Altertums IV, Graz ${ }^{2} 1961$

Sternberg-el Hotabi, H., Die Metternichstele, in: O. Kaiser (Hg.), Texte aus der Umwelt des Alten Testaments (TUAT) Bd.II/3: Rituale und Beschwörungen II, Gütersloh 1988

Vogelsang, F., Kommentar zu den Klagen des Bauern (UGAÄ 6), Leipzig 1913

Wolf-Brinkmann, E.M., Versuch einer Deutung des Begriffes $b^{\prime}$ anhand der Ueberlieferung der Fruehzeit und des Alten Reiches, Basel 1968

Zaba, Z., Les Maximes de Ptahhotep, Prag 1956

Žabkar, L.V., A Study of the Ba-Concept in Ancient Egyptian Texts, Chicago 1968 